\title{
Effect of BCG vaccination against Mycobacterium tuberculosis infection in children: systematic review and meta-analysis
}

\author{
(c) (1) $($ OPEN ACCESS
}

\begin{abstract}
A Roy senior scientist ${ }^{1}$, M Eisenhut consultant paediatrician ${ }^{2}$, R J Harris statistician ${ }^{1}$, L C Rodrigues professor of epidemiology ${ }^{3}$, S Sridhar research associate ${ }^{4}$, S Habermann junior doctor ${ }^{2}$, L Snell junior doctor ${ }^{2}$, P Mangtani senior lecturer ${ }^{3}$, I Adetifa paediatrician and medical epidemiologist ${ }^{5}$, A Lalvani professor of infectious disease ${ }^{4}$, I Abubakar professor of infectious disease epidemiology ${ }^{16}$
\end{abstract}

${ }^{1}$ Centre for Infectious Disease Surveillance and Control, Public Health England, London NW9 5EQ, UK; '2uton and Dunstable University Hospital, NHS Foundation Trust, Luton, UK; ${ }^{3}$ London School of Hygiene and Tropical Medicine, London, UK; ${ }^{4}$ Tuberculosis Research Centre, Respiratory Infections Section, National Heart and Lung Institute, Imperial College London, London, UK; ${ }^{5}$ Medical Research Council, Fajara, Gambia; ${ }^{6}$ Centre for Infectious Disease Epidemiology and MRC Clinical Trials Unit, University College London, London, UK

\begin{abstract}
Objectives To determine whether BCG vaccination protects against Mycobacterium tuberculosis infection as assessed by interferon $y$ release assays (IGRA) in children.

Design Systematic review and meta-analysis. Searches of electronic databases 1950 to November 2013, checking of reference lists, hand searching of journals, and contact with experts.

Setting Community congregate settings and households.

Inclusion criteria Vaccinated and unvaccinated children aged under 16 with known recent exposure to patients with pulmonary tuberculosis. Children were screened for infection with $M$ tuberculosis with interferon $y$ release assays.

Data extraction Study results relating to diagnostic accuracy were extracted and risk estimates were combined with random effects meta-analysis.

Results The primary analysis included 14 studies and 3855 participants. The estimated overall risk ratio was 0.81 (95\% confidence interval 0.71 to 0.92 ), indicating a protective efficacy of $19 \%$ against infection among vaccinated children after exposure compared with unvaccinated children. The observed protection was similar when estimated with the two types of interferon y release assays (ELISpot or QuantiFERON). Restriction of the analysis to the six studies ( $n=1745$ ) with information on progression to active tuberculosis at the time of screening showed protection against infection of $27 \%$ (risk ratio $0.73,0.61$ to 0.87 ) compared with $71 \%(0.29$,
\end{abstract}

0.15 to 0.58 ) against active tuberculosis. Among those infected, protection against progression to disease was $58 \%(0.42,0.23$ to 0.77$)$.

Conclusions BCG protects against $M$ tuberculosis infection as well as progression from infection to disease.

Trial registration PROSPERO registration No CRD42011001698 (www. crd.york.ac.uk/prospero/)

\section{Introduction}

BCG vaccine has been the subject of numerous efficacy trials and epidemiological studies conducted over several decades. These trials indicate that $\mathrm{BCG}$ has $60-80 \%$ protective efficacy against severe forms of tuberculosis in children, particularly meningitis, ${ }^{12}$ and its efficacy against pulmonary diseases varies geographically. ${ }^{3-5} \mathrm{BCG}$ does not seem to protect against disease when it is given to people already infected or sensitised to environmental mycobacteria, which could explain the geographical variation..$^{6-8}$ Until recently it was not possible to establish whether the protective effect of BCG vaccination against disease was from its action in preventing acquisition of infection or limited to prevention of progression from infection to clinical disease.

The scarcity of evidence on whether the vaccine is effective against Mycobacterium tuberculosis infection was because of limitations of the tuberculin skin test. This test cannot distinguish a positive response caused by $M$ tuberculosis infection from that caused by BCG vaccination or non-tuberculous 
mycobacterial infection. ${ }^{9}$ The recently developed T cell based interferon $\gamma$ release assays (IGRA) can detect $M$ tuberculosis infection and discriminate this from previous BCG vaccination and most non-tuberculous mycobacterial infections, ${ }^{10}$ allowing investigation of whether BCG protects against $M$ tuberculosis infection. ${ }^{11-13}$ If BCG is found to protect against infection, it will have key implications for its use in current immunisation programmes ${ }^{14}$ as well as in the future development of new improved tuberculosis vaccines.

In this systematic review we examined the evidence for the protective effect of BCG against $M$ tuberculosis infection, as opposed to against disease, in settings where children can be presumed to have been exposed to $M$ tuberculosis. This therefore assesses the degree to which BCG vaccination before exposure is associated with a subsequent negative result on an interferon $\gamma$ release assay. We also looked at factors that could alter the measured protective effect of BCG: latitude, the recommended age of vaccination in the area where the study was conducted, type of assay, and study quality. When there was sufficient information, we determined protection against early progression from infection to disease during screening.

\section{Methods}

\section{Search and Data Extraction}

We searched Embase (1980 until November 2013), Medline (1950 until November 2013), and the Cochrane Library as well as reference lists of retrieved articles and conference abstracts. The search strategy was not restricted by language or year. We used the following search terms, adapted for each database when appropriate: BCG, Bacillus Calmette Guerin, tuberculosis, TB, interferon $\gamma$ release assay, IGRA, ELI Spot, T-Spot TB, and QuantiFERON. We also reviewed the personal libraries of the research team. Two reviewers (AR and ME) independently screened the titles and abstracts (when available) from the search results and identified studies for assessment of full text.

Disagreements were resolved by asking the opinion of a third reviewer (IA) for resolution until consensus was reached at each stage of the screening process.

Information extracted onto pretested data forms included study details (authors, year, geographical area, study design, sample size), the population (mean age, sex, and setting), exposure (method of assessment and timing), and two outcomes measures: interferon $\gamma$ release assays (as a proxy for tuberculosis infection) and active tuberculosis (when reported). When relevant information was missing, we contacted study authors by email. For some studies, the stated primary outcome was not $M$ tuberculosis infection but data on results of interferon $\gamma$ release assays had been collected. For these studies, we contacted the authors to obtain the raw data.

\section{Inclusion and exclusion criteria}

Studies meeting the following criteria were included:

- Participants had known recent exposure to people with pulmonary tuberculosis. We assumed that in such studies, a reduced rate of infection in vaccinated versus unvaccinated children could be attributed to protection by vaccination rather than lack of exposure.

- Participants were children aged under 16, including those who had and had not been vaccinated with BCG.

- All children were screened for infection with $M$ tuberculosis with interferon $\gamma$ release assays; either in tube $\gamma$ interferon quantification (QuantiFERON) or enumeration of $\gamma$ interferon releasing T cells (ELISpot).
- We excluded all studies in which interferon $\gamma$ release assays was used only in children with positive results from tuberculin skin test or in which participants were not tested by interferon $\gamma$ release assays or when the study reported on active tuberculosis or results of tuberculin skin test only or where the study was conducted in animals.

\section{Quality assessment for individual studies}

Two reviewers (AR and ME) assessed aspects of the quality of each selected study using a modified version of the Newcastle-Ottawa scale for assessment of observational cohort studies. ${ }^{15}$ This scale awards a maximum of nine points to each study: four points for the adequate selection of participants, two points for comparability of the studies based on the design and analysis, and three points for the adequate ascertainment of outcome. We defined studies of high quality as those that scored $\geq 66.6 \%$, moderate for 33.3-66.6\%, and low for those $<33.3 \%$. Discrepancy in quality assessment was discussed and resolved by the two reviewers.

\section{Definitions}

- Exposure to tuberculosis was defined as close contact with people with active pulmonary tuberculosis as identified by the treating physicians.

- Infection was defined as any positive results of interferon $\gamma$ release assays in contacts irrespective of whether they had evidence of active tuberculosis or not. Participants with an indeterminate status were excluded from the analysis.

- Disease was defined as presence of active tuberculosis (according to the authors) with or without a positive result on interferon $\gamma$ release assays. Outcomes of both infection and active disease were identified at the point of screening for infection after exposure. A small number of additional cases might have been included as some outbreak investigations were undertaken over a period of several weeks.

\section{Synthesis of results}

\section{Primary analysis-protection against infection}

Our primary analysis focused on whether BCG vaccination administered before exposure was associated with a subsequent negative result on interferon $\gamma$ release assay in children who had contact with infectious tuberculosis. We used binary data to categorise participants into those who were or were not vaccinated with $\mathrm{BCG}$ and those who did or did not have a negative assay result (independent of progression to disease), with continuity corrections applied to zero cells. ${ }^{16}$ To include all studies in a single analysis, we combined results from studies reporting both QuantiFERON and ELIspot. Where results of both assays were available from a study we chose the QuantiFERON result as this was most used among the selected studies.

\section{Subgroup analyses of protection against infection}

We used subgroup analysis to assess whether the use of different types of interferon $\gamma$ release assay (ELISpot and QuantiFERON) led to different results. We also investigated the relation between the observed effect of BCG and age at which BCG vaccination was recommended in the area where the study was conducted (we used policy recommendations because we did not have timing of BCG vaccination in individuals); geographical latitude 
where the study was conducted: latitudes of the study location was categorised into three groups: $0-20^{\circ}, 20-40^{\circ}$, and $>40^{\circ}$; and study quality: we investigated the effect of study quality by application of the Newcastle-Ottawa scale. ${ }^{15}$ Using this scale, we compared studies with a point rating on this scale equal or above and below the median of 5 points.

\section{Secondary analysis — protection against infection protection against disease}

In a secondary analysis, we estimated protection by BCG against active disease in a subset of six studies that reported development of active tuberculosis during screening. This was achieved by examining, in this subset, the number of children with active disease (regardless of assay status) in those who received BCG versus those who did not. In the population with tuberculosis infection determined from positive result on interferon $\gamma$ release assays, we also examined the effect of BCG on progression from infection to early active disease by comparing the number with active disease in those who received BCG versus those who did not.

\section{Statistical analysis}

Analysis was performed with the DerSimonian and Laird random effects model, which incorporates variation between studies in the weighting. To quantify inconsistency across studies by describing percentage of the variability in effect estimates from heterogeneity we used the $\mathrm{I}^{2}$ statistic with $\mathrm{I}^{2}$ $>40 \%$ representing moderate, $>60 \%$ substantial, and $>80 \%$ considerable heterogeneity. ${ }^{17} \mathrm{We}$ applied a continuity correction to zero cells to include all studies in the analysis; the approach used is designed to minimise bias, ${ }^{16}$ although in our case the potential bias from any such correction would be extremely small as only one study contained zero counts and there were plenty of well powered studies. We assessed potential factors behind the heterogeneity with random effects meta-regression. ${ }^{18}$ The protective effect of BCG was described with risk ratios and $95 \%$ confidence intervals. To assess potential publication bias we used funnel plots and the Harbord test. ${ }^{19}$

\section{Results \\ Study selection}

Our literature search found 601 articles; based on a review of titles, we selected 546 abstracts for further assessment leading to the identification of 133 articles for full text review (fig 1). $\Downarrow$ After we reviewed the full text, 112 articles did not meet the inclusion criteria. The 21 remaining articles (describing 21 studies) were selected for potential inclusion. Fourteen articles stated that interferon $\gamma$ release assays were carried out but did not report the results on the relation between $\mathrm{BCG}$ vaccination and assay. The authors were contacted and asked to provide the raw data. For seven such studies, we received no response after repeated attempts. The analysis therefore included 14 studies (fig 1). $\Downarrow$

\section{Characteristics of included studies}

The meta-analysis included cohort studies of children who were screened as part of an investigation of a tuberculosis outbreak, ${ }^{11}{ }^{20-22}$ who were referred to hospital as close contacts of index cases, ${ }^{2021}{ }^{23-25}$ were household contacts of an infectious adult, ${ }^{1326-28}$ or were contacts in other settings. ${ }^{29}$ Four studies were conducted in the United Kingdom, two each in the Gambia and Spain, and a single study in Greece, Italy, Indonesia, Turkey, South Africa, and Cambodia each (table). $\Downarrow$ BCG vaccination status was assessed in included studies by one or a combination of BCG scar, ${ }^{12} 1322-272930$ confirmed medical records, ${ }^{11} 122130$ and parental recall of vaccination. ${ }^{21}$

In 13 of the 14 studies patients in the index cases had smear positive active tuberculosis. In one additional study the index case was a patient with smear negative pulmonary tuberculosis. This was a 9 year old child in whom the duration of exposure of contacts at school was for a long unspecified period of time. ${ }^{11}$

\section{Primary analysis}

Our primary analysis with 14 studies and 3855 participants estimated an overall risk ratio of 0.81 (95\% confidence interval 0.71 to 0.92 , random effects estimate, fig 2 ) $\Downarrow$, indicating $19 \%$ protective efficacy against tuberculosis infection among vaccinated children after exposure compared with unvaccinated children. We noted moderate heterogeneity $\left(\mathrm{I}^{2}=40 \%, \mathrm{P}<0.06\right)$ between the studies. All studies included non-immunocompromised populations, with low probability that specific cut off values are required. The observed protection was similar for the two types of assay: ELISpot (risk ratio 0.83, 95\% confidence interval 0.68 to 1.02 ; four studies) and QuantiFERON (0.78, 0.64 to $0.96 ; 10$ studies) (fig 3). $\Downarrow$

Eight of the 10 Quantiferon studies used Quantiferon In Tube Gold, which includes a further antigen (TB7.7). Further stratification by ELISpot, Quantiferon, and Quantiferon In Tube showed no evidence of inconsistency (data not shown). In eight studies, the cut off for positive assay result was $0.35 \mathrm{IU} / \mathrm{mL}$ or they reported using the manufacturer's; one used a cut off of $10 \mathrm{IU} / \mathrm{mL}^{29}$ and one provided no information-omission of this study does not alter the conclusion.

\section{Subgroup analysis: variables affecting protection against infection}

Figure 4 shows a summary of the subgroup analysis by study characteristics. $\Downarrow$ Within subgroups for latitude there was moderate heterogeneity; and we found greater protection at higher latitudes, although the difference in protection by latitude was not significant in meta-regression ( $\mathrm{P}=0.56$, fig 4$)$. $\Downarrow$ Studies conducted above $40^{\circ}$ latitude showed a protective efficacy of BCG vaccination of $26 \%$ (risk ratio $0.74,95 \%$ confidence interval 0.60 to 0.91 ), while studies conducted at lower latitudes of $20-40^{\circ}(0.88,0.54$ to 1.45$)$ and $20-0^{\circ}(0.87,0.72$ to 1.04$)$ (fig A, appendix 1) showed no evidence of a protective effect.

Subgroup analysis comparing areas according to recommended age of BCG vaccination (fig B, appendix 1) showed no evidence for a difference in efficacy according to vaccination policy: where the policy was not to vaccinate at birth, protection by BCG against $M$ tuberculosis infection was effective (risk ratio $0.72,95 \%$ confidence interval 0.56 to 0.93 ); and in other areas it was not as effective $(0.85,0.73$ to 0.98$)$, with meta-regression giving a $\mathrm{P}$ value of 0.27 . It was difficult to distinguish between effects of vaccination policy and region.

\section{Exposure to tuberculosis}

About half (48\%) of the children ( $\mathrm{n}=1862$, five studies ${ }^{11-132021}$ ) included were exposed to a single well defined source simultaneously or it was known that there was no difference in exposure. ${ }^{13}$ A subgroup analysis of these studies found a higher vaccine efficacy of $28 \%$ (risk ratio of $0.73,95 \%$ confidence interval 0.52 to 1.00$)$. 


\section{Study quality}

Based on a modified Newcastle-Ottawa scale, we considered three studies to be of high quality, eight of moderate quality, and three of low quality (table $\Downarrow$ and see appendix 2 for details). Five articles provided information on degree of exposure to infectious cases. ${ }^{11-1327} 29$ Three articles reported that BCG vaccination was given at birth, ${ }^{132}{ }^{27}$ while the remaining articles did not provide time of vaccination. Two of the articles provided precise information on the timing of interferon $\gamma$ release assay relative to presumed exposure to an infectious case. ${ }^{12}{ }^{27}$ Three of the 14 studies included stated that the association between BCG vaccination and a negative assay result was primary or secondary outcome. ${ }^{11-13}$ The study question in Soysal and colleagues was whether BCG vaccination affects the risk of infection in children exposed to tuberculosis. ${ }^{13}$ The study of Eisenhut and colleagues was carried out during a school outbreak $^{11}$ and the study by Eriksen and colleagues during a nursery outbreak ${ }^{12}$; in both the primary aim was to assess whether BCG vaccination had a protective effect against $M$ tuberculosis infection. In two of the articles included the investigators were blinded. ${ }^{13}{ }^{22}$ In one study, clinical diagnosis of tuberculosis was made without the assay results, and the researchers in the laboratory did not see the clinical data before the tests were carried out. ${ }^{22}$ In the second study, people who performed and read the assays were blind to all personal identifiers and results of tuberculin skin tests. ${ }^{13}$ Most studies $(n=10)$ were able to show adequate follow-up of exposed children until the interferon $\gamma$ release assays, with few or no children lost to follow-up between presumed exposure to an infectious case and application of the assay.

We compared studies with a quality rating of 3-4 with those with a rating of $\geq 5$ (fig $C$, appendix 1). Studies with a rating of $\geq 5$ showed protection (risk ratio $0.68,95 \%$ confidence interval 0.55 to 0.84$)$ and those with a rating of 3 or 4 didn't $(0.88,0.77$ to 1.01 ); ( $\mathrm{P}$ value for difference 0.06 ; fig 4$) \Downarrow$. This explained $16.4 \%$ of heterogeneity between studies (residual heterogeneity $\mathrm{I}^{2}=24 \%$ )

\section{Publication bias assessment}

The funnel plot in figure $5 \Downarrow$ showed no evidence of publication bias, and the Harbord test gave a $\mathrm{P}$ value of 0.69$).{ }^{19}$ One study was dropped from this analysis because of a number of cells in the table with zero observations. ${ }^{21}$

\section{Secondary analysis-protection against infection $v$ protection against disease}

We conducted an analysis of six studies that reported the number of people who progressed to active tuberculosis disease during screening. ${ }^{11-132327} 29$ This additional information allowed us to estimate the protective effect of BCG immunisation against infection; the overall protective effect against active tuberculosis; and the protective effect against progression from $M$ tuberculosis infection to active disease (fig 6) $\Downarrow$. Interferon $\gamma$ release assay status was not considered (whether performed or not) when we calculated the number of those who developed active tuberculosis within the population. In these six studies, the protection against infection (in all children, irrespective of whether they went on to develop disease) was $27 \%$ (risk ratio $0.73,95 \%$ confidence interval 0.61 to 0.87 ; fig $7 \Downarrow$ ) and protection against active tuberculosis was $71 \%(0.29,0.15$ to 0.58). Protection by BCG against progression from infection to active disease, calculated by using infected individuals, was $58 \%(0.42,0.23$ to 0.77 ; fig 6 and fig 7$)$. $\Downarrow \Downarrow$

\section{Discussion \\ Principal findings}

This systematic review shows that BCG vaccination can protect against $M$ tuberculosis infection and consolidates the results from recent studies showing evidence of protection against infection. ${ }^{11-1329}$ Our results support a paradigm shift in the understanding of how antimycobacterial vaccines (new and old) can work, from the view that BCG protects against disease but not against infection to one that it protects against infection itself. $^{31}$

A recent double blind randomised placebo controlled phase $2 b$ trial of a BCG prime-MVA85A boost vaccination in infants showed no evidence of additional protection, over and above that of BCG against tuberculosis disease infection. ${ }^{32}$ This is despite MVA85A inducing a significantly higher Ag85-specific CD4 positive T cell expression of interferon $\gamma, \mathrm{TNF} \alpha$, and interleukin 2 compared with placebo. One possible explanation for this lack of increased efficacy might be that BCG conferred protection against $M$ tuberculosis infection was sufficiently high such that an additional immunisation with MVA85A was unable to confer additional protection.

The observed protection against $M$ tuberculosis infection was independent of the assay method used for measurement. There was no evidence of protection against infection less than $40^{\circ}$ latitude away from the equator, although the number of studies was small and the difference was not significant. If this finding is confirmed, then the effect of latitude would be consistent with the variation in protection by BCG against pulmonary disease. ${ }^{8}$ In this case, the mechanism here could be the same: that the higher prevalence of non-tuberculous mycobacterial infection close to the equator decreases the measured protection with BCG. The limited number of studies reviewed from locations closer to the equator, however, means that firm conclusions must wait until new studies find similar results.

High quality studies also showed protection by BCG vaccination against infection, while lower quality studies did not suggest that study design factors in smaller low quality studies led to the inability to detect a protective effect. This has been observed in systematic reviews of trials of BCG vaccination against tuberculosis. $^{8}$

We found evidence of progression to active tuberculosis as identified at point of screening of index cases. Our interpretation of progression at the time of screening potentially includes both early and late progressors because of uncertainty of the timing of progression to active disease. Publication of an analysis of follow-up data from a study used in this meta-analysis ${ }^{13}$ recently reported protective efficacy of the vaccine of $67 \%$ for protection against progression from infection to active disease. ${ }^{33}$ Similar longitudinal cohorts and randomised control trials will provide a better understanding of the protective efficacy of BCG vaccination

\section{Limitations and strengths}

Our results should be treated with caution for several reasons. Data were not always complete, and we could not control for, or explore interaction with, all variables. For instance, it was assumed there was no prior infection before the documented exposure to active disease or outbreak. Such exposure, however, should affect vaccinated and unvaccinated children equally. Vaccinated children are unlikely to be different from unvaccinated children in other ways that could contribute to protection from infection. We attempted to lower the probability 
of prior exposure by restricting the analysis to children aged under 16.

We tried to minimise bias from differences in exposure in the two groups by limiting the review to studies among contacts - that is, to outbreaks among contacts of infectious cases or known household exposure where exposure is likely to be similar in vaccinated and unvaccinated children. It is important, however, to consider the possibility that studies included in this review had vaccine recipients who were less likely to be exposed to infection before the outbreak. Exposure can differ because of variable duration of infectiousness of the index patient and the amount of effective contact.

Reduced infection rates in children immunised with BCG could also be caused by the fact that parents who are generally more aware of infection control measures are from higher educated families with lower exposure to $M$ tuberculosis infection and have their children more comprehensively immunised, which includes BCG immunisation. A further potential source of bias relates to the greater likelihood that private paediatricians who care for children from medium and high socioeconomic levels might recommend against BCG vaccination if they consider these children to be at a lower risk of infection. We included various observational studies from settings with different socioeconomic backgrounds, potentially neutralising such bias. We also acknowledge that further quantification of degree of exposure to tuberculosis cases, including proximity to the index case and the number of cases in close contact, was not possible in our study because the primary studies were not designed to compare exposure in children who were and were not immunised with BCG.

Investigation of the effect of age at vaccination at an individual level and of time from vaccination to exposure to an infectious case would be of particular interest. The use of interferon $\gamma$ release assays as an indicator of infection is still subject to some debate, although this is currently the best marker for infection and certainly discriminates well between infection with $M$ tuberculosis and previous BCG vaccination.

There are suggestions that different strains of BCG can induce a broad range of immunological response, which in turn brings about a change in the level of protection. A recent meta-analysis of trials, including 18 studies reporting on protection against pulmonary and six reporting on protection against miliary or meningeal tuberculosis, showed no evidence that efficacy of BCG was associated with vaccination strains. ${ }^{8}$ These results are consistent with results from the UKMRC trials, which found equivalent protection by the Copenhagen strain of BCG and an $M$ microti derived vaccine. ${ }^{6}$ Only three ${ }^{11327}$ of the 14 studies included in our review provided information on the $B C G$ vaccine strains used, so we also used the UK studies ${ }^{11-1320} 2127$ from which we had information on the strains of BCG used in British school children and found no evidence of bias by vaccine strain (data not shown).

We limited subgroup analyses to investigate heterogeneity because of the potential for spurious findings. Where assessed, the main differences in the subgroup analyses were for rating on the Newcastle-Ottawa scale, the P value for which was similar under the permutation test recommended by Higgins and Thompson. ${ }^{17}$ Similarly, the small number of studies limited the power to explore whether the variation in protection by $\mathrm{BCG}$ against tuberculosis is mostly through variation in protection against infection. The primary aim of most $(75 \%)$ studies reviewed was not to investigate $\mathrm{BCG}$ protection against infection. This, taken in combination with our symmetrical funnel plot, suggests that our findings are unlikely to be caused by publication or reporting bias.

\section{Implications and conclusions}

The effect of BCG vaccination, and its policy implications, would be different for countries with high versus low incidence of tuberculosis especially in terms of the cost effectiveness of policies. Countries with a policy of vaccination at birth tend to be those countries with a higher incidence of tuberculosis (cut off $>20 / 100000$ according to European Centre for Disease Prevention (ECDC)) than those countries without such vaccination policy $(<20 / 100000)$. Given the incomplete control of tuberculosis, especially in high burden countries, optimisation of use of BCG is sensible. Our analysis supports vaccinating children as soon as possible after birth to prevent infection and disease.

Our results provide evidence that BCG protects against tuberculosis infection from multiple epidemiologically different settings and independent of the type of interferon $\gamma$ release assay used to detect infection. Future trials of candidate vaccines need to investigate the efficacy of the new vaccine against tuberculosis infection and early progression and late progression to active disease. In addition, transparent reporting from outbreak and contact studies of individual ages and time of vaccination and exposure to tuberculosis of participants should allow future meta-analysis to investigate protection against infection. Our results also suggest that models of BCG impact should be revised to include an effect against infection and consequently latent $M$ tuberculosis infection and reactivation as without this the effect of BCG would be underestimated.

Contributors: AR and ME contributed equally to the work. IA, ME, and AR conceived idea and designed the study. AR and ME acquired the data. SH and LS contributed to the literature search and quality assessment. RJH was responsible for the statistical analysis. RJH, IA, ME, AR, PM, LCR, and AL analysed and interpreted the data. ME and AR drafted the manuscript. All authors contributed to planning, interpretation and writing of the manuscript and critically revised the manuscript for intellectual content. IA is guarantor.

Funding: This research received no specific grant from any funding agency in the public, commercial, or not-for-profit sectors. IA is supported by the UK National Institute for Health Research and the UK MRC.

Competing interests: All authors have completed the ICMJE uniform disclosure form at www.icmje.org/coi_disclosure.pdf and declare: no support from any organisation for the submitted work; $A L$ is inventor for several patents underpinning $T$ cell based diagnosis. The ESAT-6/CFP-10 IFN-gamma ELISpot was commercialised by an Oxford University spin-out company (T-SPOT.TB, Oxford Immunotec, Abingdon, UK), in which Oxford University and AL have minority shares of equity and entitlement to royalties.

Ethical approval: Not required.

Declaration of transparency: IA affirms that the manuscript is an honest, accurate, and transparent account of the study being reported; that no important aspects of the study have been omitted; and that any discrepancies from the study as planned (and, if relevant, registered) have been explained.

Data sharing: Technical appendix, statistical code, and dataset are available from IA.

Rodrigues LC, Mangtani P, Abubakar I. How does the level of BCG vaccine protection against tuberculosis fall over time? BMJ 2011;343:d5974.

2 Trunz BB, Fine P, Dye C. Effect of BCG vaccination on childhood tuberculous meningitis and miliary tuberculosis worldwide: a meta-analysis and assessment of cost-effectiveness. Lancet 2006;367:1173-80. 


\section{What is already known on this topic}

Numerous efficacy trials indicate that BCG has $60-80 \%$ protective efficacy against severe forms of tuberculosis in children, particularly meningitis; its efficacy when given to adults against pulmonary diseases varies geographically probably because of previous infection when BCG is given

The recently developed $T$ cell based interferon $y$ release assays can detect $M$ tuberculosis infection and discriminate this from previous BCG vaccination and most non-tuberculous mycobacterial infections, allowing investigation of whether BCG protects against $M$ tuberculosis infection

\section{What this study adds}

In addition to protection against tuberculosis disease, BCG protects against tuberculosis infection

A protective effect against tuberculosis infection was observed from multiple epidemiologically different settings and independent of the type of interferon $y$ release assay used to detect infection

3 Colditz GA, Brewer TF, Berkey CS, Wilson ME, Burdick E, Fineberg HV et al. Efficacy of $B C G$ vaccine in the prevention of tuberculosis. Meta-analysis of the published literature. JAMA 1994:271:698-702.

4 Lienhardt C, Zumla A. BCG: the story continues. Lancet 2005;366:1414-6.

5 Zodpey SP, Shrikhande SN. The geographic location (latitude) of studies evaluating protective effect of BCG vaccine and it's efficacy/effectiveness against tuberculosis. Indian $J$ Public Health 2007;51:205-10.

6 Abubakar I, Pimpin L, Ariti C, Beynon R, Mangtani P, Sterne JA, et al Systematic review and meta-analysis of the current evidence on the duration of protection by bacillus Calmette-Guerin vaccination against tuberculosis. Health Technol Assess 2013;17:1-371.

$7 \quad$ Narayanan PR. Influence of sex, age and nontuberculous infection at intake on the efficacy of BCG: re-analysis of 15-year data from a double-blind randomized control trial in South India. Indian J Med Res 2006;123:119-24.

8 Mangtani P, Abubakar I, Ariti C, Mangtani P, Abubakar I, Ariti C et al. Protection by BCG against tuberculosis: a systematic review of randomised controlled trials. Clin Infect Dis 2013;58:470-80.

9 Fine PE. BCG vaccination against tuberculosis and leprosy. Br Med Bull 1988;44:691-703.

10 Lalvani A, Millington KA. T cell-based diagnosis of childhood tuberculosis infection. Curr Opin Infect Dis 2007;20:264-71.

11 Eisenhut M, Paranjothy S, Abubakar I, Bracebridge S, Lilley M, Mulla R, et al. BCG vaccination reduces risk of infection with Mycobacterium tuberculosis as detected by gamma interferon release assay. Vaccine 2009;27:6116-20.

12 Eriksen J, Chow JY, Mellis V, Whipp B, Walters S, Abrahamson E, et al. Protective effect of $B C G$ vaccination in a nursery outbreak in 2009: time to reconsider the vaccination threshold? Thorax 2010;65:1067-71.

13 Soysal A, Millington KA, Bakir M, Dosanjh D, Aslan Y, Deeks JJ, et al. Effect of BCG vaccination on risk of Mycobacterium tuberculosis infection in children with household tuberculosis contact: a prospective community-based study. Lancet 2005;366:1443-51.

14 Abu-Raddad LJ, Sabatelli L, Achterberg JT, Sugimoto JD, Longini IM Jr, Dye C, et al. Epidemiological benefits of more-effective tuberculosis vaccines, drugs, and diagnostics. Proc Natl Acad Sci U S A 2009;106:13980-5.

15 Wells GA, Shea B, O'Connell D, Peterson J, Welch V, Losos M, et al. The Newcastle-Ottawa Scale (NOS) for assessing the quality if nonrandomized studies in meta-analyses. www.ohri.ca/programs/clinical_epidemiology/oxford.asp.

16 Sweeting MJ, Sutton AJ, Lambert PC. What to add to nothing? Use and avoidance of continuity corrections in meta-analysis of sparse data. Stat Med 2004;23:1351-75.

17 Higgins JP, Thompson SG. Controlling the risk of spurious findings from meta-regression. Stat Med 2004;23:1663-82.

18 Higgins JPT, Green S, eds. Cochrane handbook for systematic reviews of interventions. John Wiley, 2011.

19 Harbord RM, Egger M, Sterne JA. A modified test for small-study effects in meta-analyses of controlled trials with binary endpoints. Stat Med 2006;25:3443-57.

20 Ewer K, Deeks J, Alvarez L, Bryant G, Waller S, Andersen P, et al. Comparison of T-cell-based assay with tuberculin skin test for diagnosis of Mycobacterium tuberculosis infection in a school tuberculosis outbreak. Lancet 2003:361:1168-73.

21 Neira-Munoz E, Smith J, Cockcroft P, Basher D, Abubakar I. Extensive transmission of Mycobacterium tuberculosis among children on a school bus. Pediatr Infect Dis J 2008;27:835-7
22 Altet-Gomez N, De Souza-Galvao M, Latorre I Milà C, Jiménez MA, Solsona J, et al. Diagnosing TB infection in children: analysis of discordances using in vitro tests and the tuberculin skin test. Eur Respir J 2011;37:1166-74

23 Dominguez J, Ruiz-Manzano J, De Souza-Galvao M, De Souza-Galvão M, Latorre I, Milà $\mathrm{C}$, et al. Comparison of two commercially available gamma interferon blood tests for immunodiagnosis of tuberculosis. Clin Vaccine Immunol 2008;15:168-71.

24 Hill PC, Brookes RH, Adetifa IM, Fox A, Jackson-Sillah D, Lugos MD, et al. Comparison of enzyme-linked immunospot assay and tuberculin skin test in healthy children exposed to Mycobacterium tuberculosis. Pediatrics 2006;117:1542-8.

25 Rutherford ME, Nataprawira M, Yulita I, Sampurno H, Ruslami R. QuantiFERON(R)-TB Gold In-Tube assay vs. tuberculin skin test in Indonesian children living with a tuberculosis case. Int J Tuberc Lung Dis 2012;16:496-502.

26 Adetifa IM, Ota MO, Jeffries DJ, Hammond A, Lugos MD, Donkor S, et al Commercial interferon gamma release assays compared to the tuberculin skin test for diagnosis of latent Mycobacterium tuberculosis infection in childhood contacts in the Gambia. Pediatr Infect Dis J 2010;29:439-43.

27 Okada K, Mao TE, Mori T, Miura T, Sugiyama T, Yoshiyama T, et al. Performance of an interferon-gamma release assay for diagnosing latent tuberculosis infection in children. Epidemiol Infect 2008;136:1179-87.

28 Tsiouris SJ, Austin J, Toro P, Coetzee D, Weyer K, Stein Z, et al. Results of a tuberculosis-specific IFN-gamma assay in children at high risk for tuberculosis infection. Int J Tuberc Lung Dis 2006;10:939-41.

29 Tsolia MN, Mavrikou M, Critselis E, Papadopoulos NG, Makrinioti H, Spyridis NP, et al. Whole blood interferon-gamma release assay is a useful tool for the diagnosis of tuberculosis infection particularly among Bacille Calmette Guerin-vaccinated children. Pediatr Infect Dis J 2010;29:1137-40.

30 Bianchi L, Galli L, Moriondo M, Veneruso G, Becciolini L, Azzari C, et al. Interferon-gamm release assay improves the diagnosis of tuberculosis in children. Pediatr Infect Dis $J$ 2009;28:510-4.

31 Lalvani A, Sridhar S. BCG vaccination: 90 years on and still so much to learn. Thorax 2010;65:1036-8.

32 Tameris MD, Hatherill M, Landry BS, Scriba TJ, Snowden MA, Lockhart S, et al; MVA85A 020 Trial Study Team. Safety and efficacy of MVA85A, a new tuberculosis vaccine, in infants previously vaccinated with BCG: a randomised, placebo-controlled phase $2 b$ trial. Lancet 2013;381:1021-8.

33 Dosanjh DP, Bakir M, Millington KA, Soysal A, Aslan Y, Efee S, et al. Novel M tuberculosis antigen-specific T-cells are early markers of infection and disease progression. PLOS One 2011;6:e28754.

\section{Accepted: 11 July 2014}

\section{Cite this as: BMJ 2014;349:g4643}

This is an Open Access article distributed in accordance with the Creative Commons Attribution Non Commercial (CC BY-NC 3.0) license, which permits others to distribute, remix, adapt, build upon this work non-commercially, and license their derivative works on different terms, provided the original work is properly cited and the use is non-commercial. See: http://creativecommons.org/licenses/by-nc/3.0/. 


\section{Table}

Table 1/ Characteristics of studies included in systematic review of effect of BCG vaccination against mycobacterium tuberculosis infection in children

\begin{tabular}{|c|c|c|c|c|c|c|}
\hline & Study period & Country & Setting & $\begin{array}{l}\text { No of } \\
\text { participants }\end{array}$ & Age group (years) & Quality rating* \\
\hline Adetifa 2010 & NS & Gambia & Childhood household contacts & $236 \dagger$ & 6 months- 14 & Moderate \\
\hline Altet-Gomez 2011 & Sept 2005-Sept 2007 & Spain & $\begin{array}{l}\text { Contacts with smear positive active TB } \\
\text { patient }\end{array}$ & 166 & $<15$ & Moderate \\
\hline Bianchi 2009 & $\begin{array}{l}1 \text { July 2005-31 December } \\
2006\end{array}$ & Italy & $\begin{array}{l}\text { Referred to hospital as close contacts of } \\
\text { index cases }\end{array}$ & $18 \dagger$ & $<16$ & Moderate \\
\hline Domingues 2008 & Sept 2004-Nov 2006 & Spain & $\begin{array}{l}\text { Individuals registered as close contacts of } \\
\text { patients }\end{array}$ & 117 & $0-16$ & Moderate \\
\hline Eisenhut 2009 & 2007 & UK & Outbreak investigation & 199 & $7-10$ & Moderate \\
\hline Eriksen 2010 & 2009 & UK & Outbreak investigation-nursery & 126 & $0-9$ & High \\
\hline Ewer 2003 & 2001 & UK & School outbreak study & 535 & $11-15$ & Moderate \\
\hline Hill 2006 & $\begin{array}{l}\text { June 2002-September } \\
2004\end{array}$ & Gambia & $\begin{array}{l}\text { Child contacts of patient with sputum smear } \\
\text { positive tuberculosis }\end{array}$ & $643 \dagger$ & $0-14$ & Low \\
\hline Okada 2008 & 2005 & Cambodia & $\begin{array}{l}\text { Young children living with pulmonary TB } \\
\text { patients }\end{array}$ & $195 \dagger$ & $0-5$ & Moderate \\
\hline Rutherford 2012 & NS & Indonesia & $\begin{array}{l}\text { Exposed to household and neighbourhood } \\
\text { index cases }\end{array}$ & 330 & 9 months -9 & Low \\
\hline Soysal 2005 & October 2002-April 2003 & Turkey & $\begin{array}{l}\text { Child household contacts of adults with smear } \\
\text { positive pulmonary tuberculosis }\end{array}$ & 979 & $0-16$ & High \\
\hline Neira-Munoz 2008 & 2007 & UK & $\begin{array}{l}\text { School bus passengers (driver of bus was } \\
\text { index case) }\end{array}$ & 23 & Median 13 & High \\
\hline Tsiouris 2006 & NS & South Africa & Household contacts of index cases & $159 \dagger$ & $5-15$ & Moderate \\
\hline Tsolia 2010 & $\begin{array}{l}1 \text { Jan 2007-31 December } \\
2008\end{array}$ & Greece & $\begin{array}{l}\text { Contacts with adult with TB who attended TB } \\
\text { clinic }\end{array}$ & $129 \dagger$ & $<15$ & Low \\
\hline
\end{tabular}

NS=not specified.

*Newcastle-Ottawa scale rating: high $>66.6 \%$; moderate $33.3-66.6 \%$; low $<33.3 \%$.

†Data received from author. 


\section{Figures}

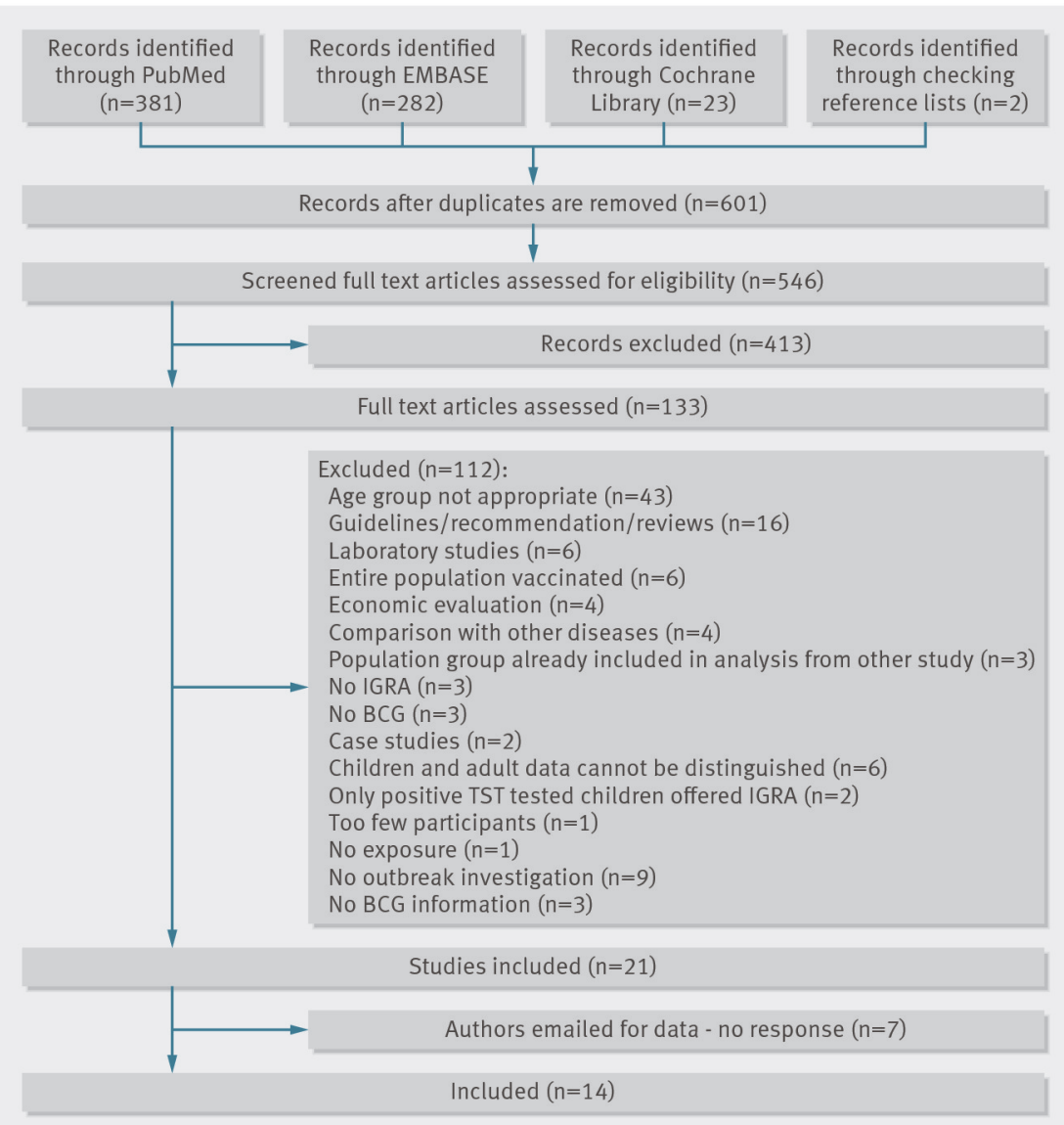

Fig 1 Study selection for systematic review of BCG vaccination against mycobacterium tuberculosis infection in children

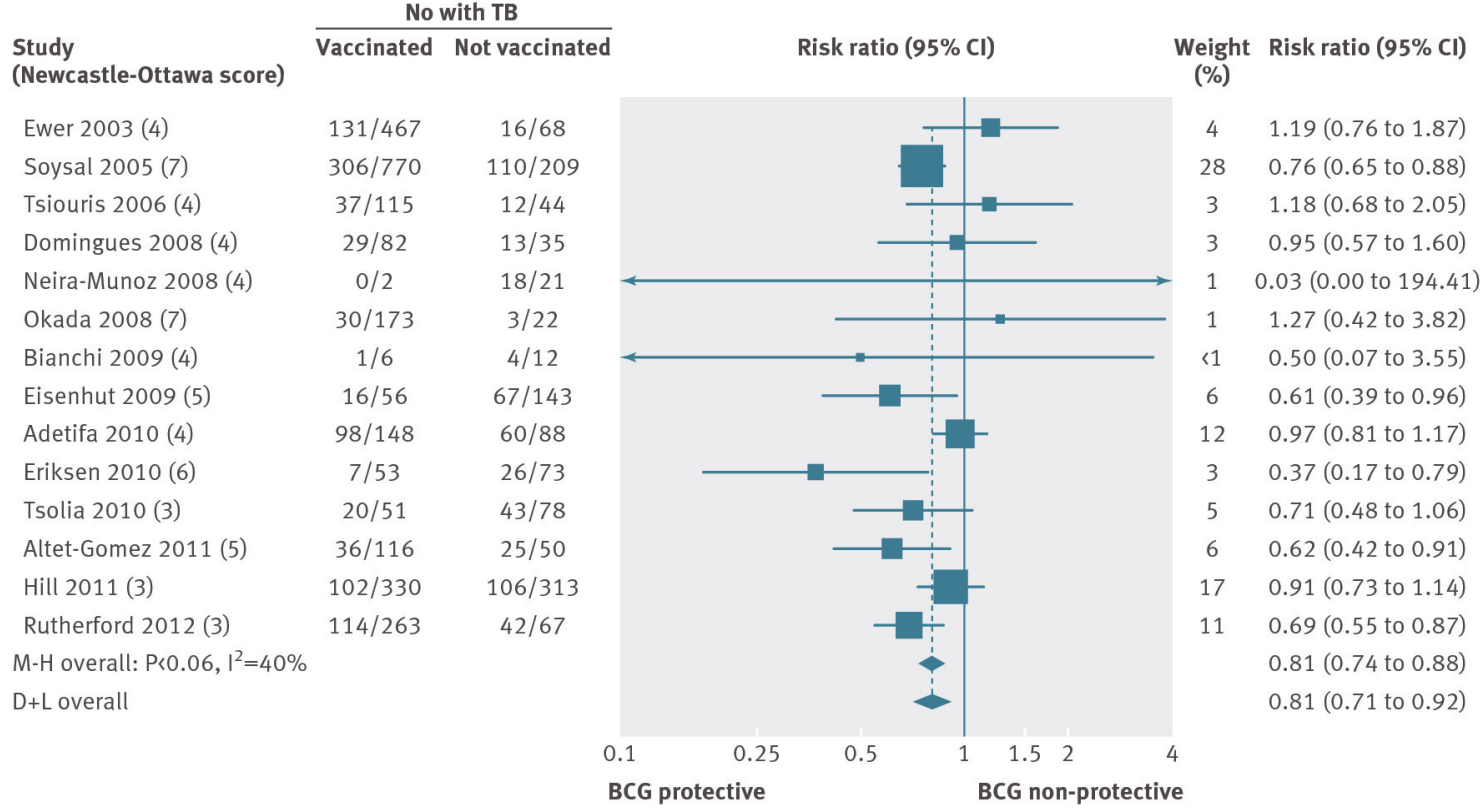

Fig 2 Protection against Mycobacterium tuberculosis infection (TB) as determined by interferon $y$ release assay (QuantiFERON) in children vaccinated with BCG. D+L=DerSimonian and Laird method; M-H=Mantel-Haenszel method. One test was used per paper and in cases where both ELISpot and QuantiFERON data were available data from QuantiFERON testing were used 


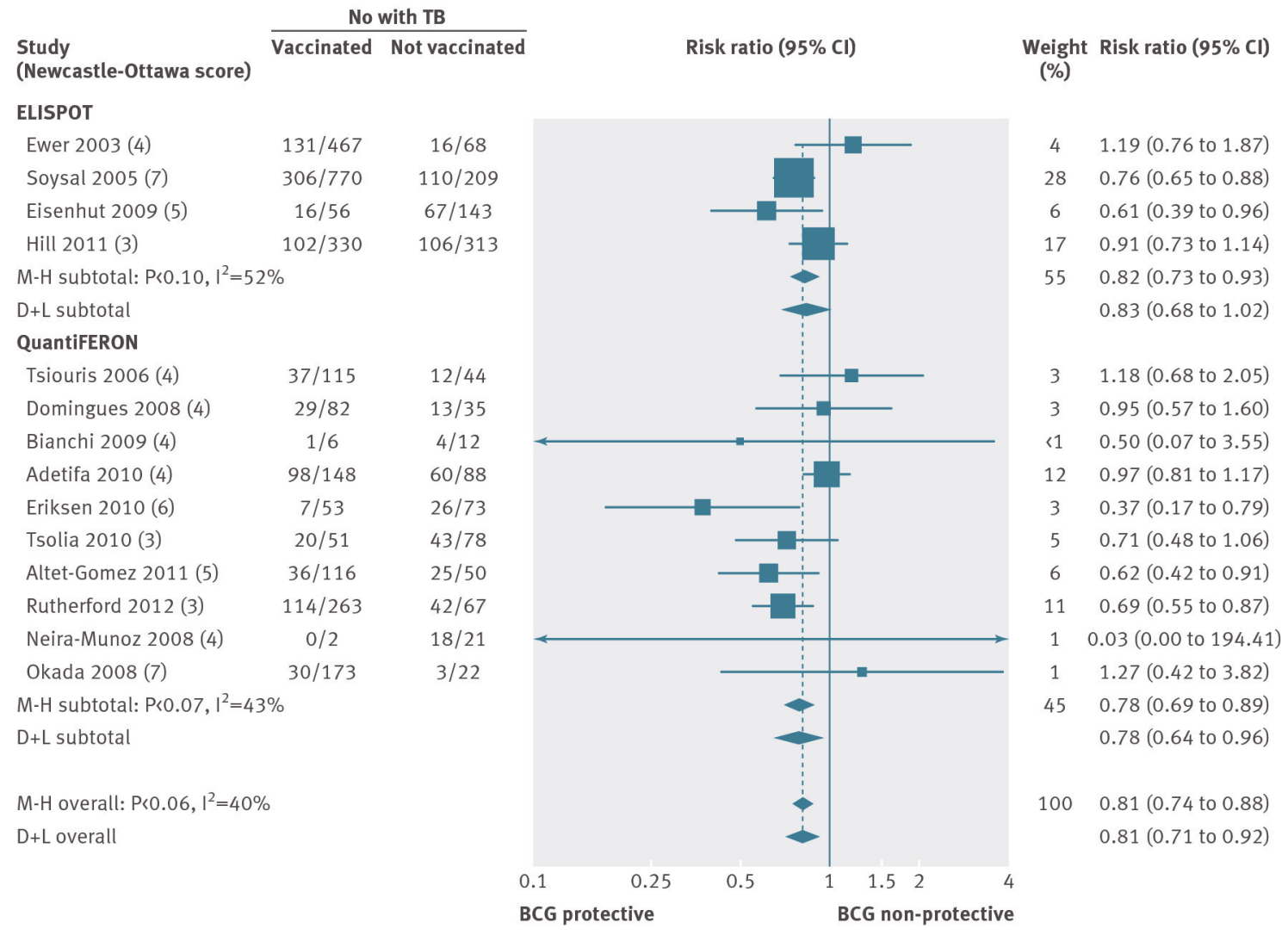

Fig 3 Protection against Mycobacterium tuberculosis infection (TB) as determined by interferon y release assay (ELISpot $v$ QuantiFERON) in children vaccinated with BCG. D+L=DerSimonian and Laird; M-H=Mantel-Haenszel

\begin{tabular}{|c|c|c|c|c|}
\hline \multirow{2}{*}{$\begin{array}{l}\text { Analysis } \\
\text { All studies }\end{array}$} & \multirow{2}{*}{$\begin{array}{c}\text { No of studies } \\
14\end{array}$} & Risk ratio $(95 \% \mathrm{Cl})$ & $\begin{array}{l}1^{2} \\
(\%)\end{array}$ & Risk ratio $(95 \% \mathrm{Cl})$ \\
\hline & & $\rightarrow$ & 40 & 0.81 (0.71 to 0.92$)$ \\
\hline \multicolumn{5}{|l|}{ Type of test $(P=0.85)$} \\
\hline ELISPOT & 4 & & 52 & 0.83 (0.68 to 1.02$)$ \\
\hline QuantiFERON & 10 & & 43 & $0.78(0.64$ to 0.96$)$ \\
\hline \multicolumn{5}{|l|}{ Latitude $(P=0.56)$} \\
\hline$<20$ & 4 & & 49 & $0.87(0.72$ to 1.04$)$ \\
\hline $20-40$ & 2 & & 54 & 0.88 (0.54 to 1.45$)$ \\
\hline$>40$ & 8 & - & 34 & 0.74 (0.60 to 0.91$)$ \\
\hline \multicolumn{5}{|c|}{ Newcastle-Ottawa score $(P=0.06)$} \\
\hline$<5^{\star}$ & 9 & & 23 & 0.88 (0.77 to 1.01$)$ \\
\hline$\geq 5^{*}$ & 5 & - & 28 & $0.68(0.55$ to 0.84$)$ \\
\hline \multicolumn{5}{|l|}{ Policy $(P=0.27)$} \\
\hline A & 6 & & 47 & 0.85 (0.73 to 0.98$)$ \\
\hline B & 8 & & 34 & 0.72 (0.56 to 0.93$)$ \\
\hline \multicolumn{5}{|l|}{ TB type } \\
\hline Active & 6 & & 42 & 0.29 (0.15 to 0.58$)$ \\
\hline Infection & 6 & - & 16 & $0.73(0.61$ to 0.87$)$ \\
\hline Infection to active & 6 & $\rightarrow-$ & 53 & $0.42(0.23$ to 0.77$)$ \\
\hline & & 0.5 & & \\
\hline
\end{tabular}

Fig 4 Subgroup analyses of protection in children by BCG vaccination against Mycobacterium tuberculosis infection (TB) in children vaccinated with BCG, as determined by type of interferon $y$ release assay, latitude, study quality, and age at vaccination policy comparing areas with $(A)$ and without $(B)$ policy to vaccinate infants at birth; and separate analysis of protection against active disease and against progression from infection to disease in subset of studies (DerSimonian and Laird method) 


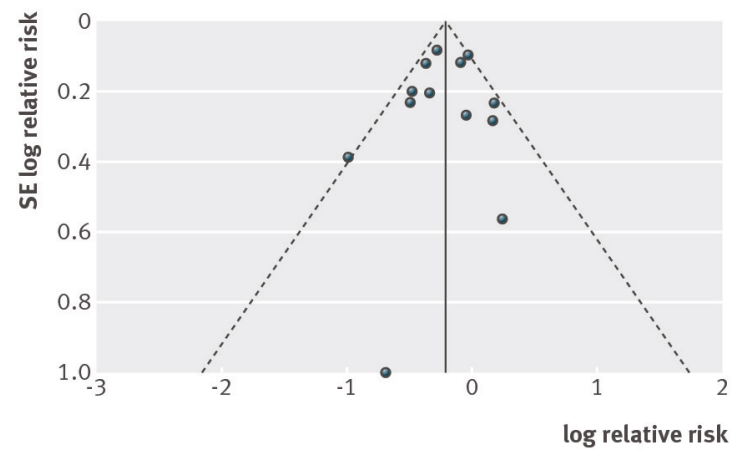

Fig 5 Funnel plot (with pseudo confidence intervals) of studies investigating association between BCG vaccination and result of interferon $y$ release assay

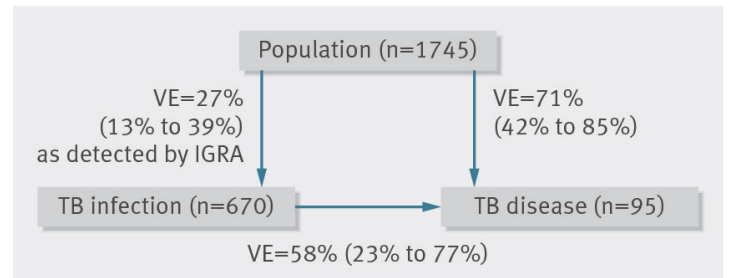

Fig 6 Schematic representation of protective effect of BCG immunisation against Mycobacterium tuberculosis infection, active tuberculosis, and progression to active tuberculosis from infection during screening from subset of six articles with data on active tuberculosis (vaccine efficacy (VE) and 95\% confidence intervals) 
No with TB

Vaccinated Not vaccinated

Risk ratio $(95 \% \mathrm{Cl})$

Infection

Soysal 2005

Domingues 2008

Okada 2008

Eisenhut 2009

Eriksen 2010

Tsolia 2010

M-H subtotal: $P=0.31,\left.\right|^{2}=16 \%$

D+L subtotal

Active TB

Soysal 2005

Domingues 2008

Okada 2008

Eisenhut 2009

Eriksen 2010

Tsolia 2010

M-H subtotal: $P=0.12,\left.\right|^{2}=42 \%$

D+L subtotal

Infection to active TB

Soysal 2005

Domingues 2008

Okada 2008

Eisenhut 2009

Eriksen 2010

Tsolia 2010

M-H subtotal: P<0.06, $I^{2}=53 \%$

D+L subtotal

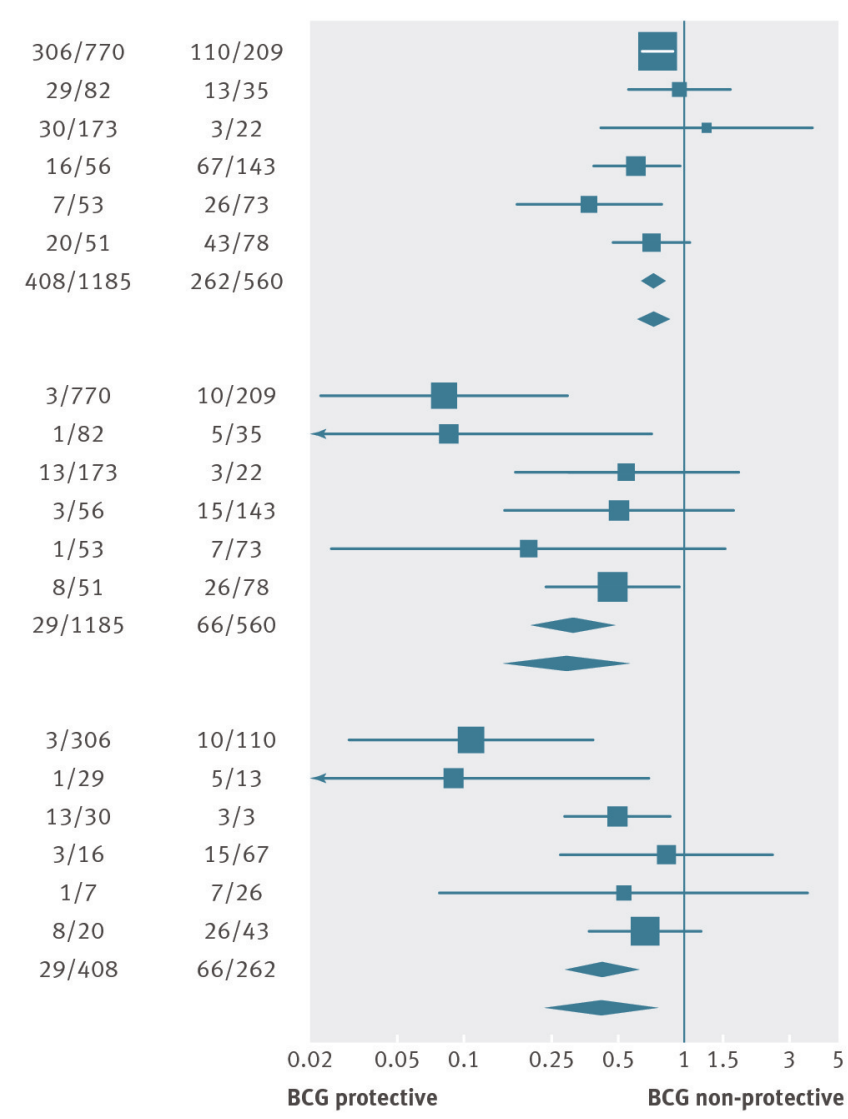

Weight Risk ratio $(95 \% \mathrm{Cl})$

(\%)

$60 \quad 0.76(0.65$ to 0.88$)$

$0.95(0.57$ to 1.60$)$

1.27 (0.42 to 3.82$)$

$0.61(0.39$ to 0.96$)$

0.37 (0.17 to 0.79 )

0.71 (0.48 to 1.06$)$

$100 \quad 0.72$ (0.63 to 0.83 )

0.73 (0.61 to 0.87$)$

0.08 (0.02 to 0.29)

0.09 (0.01 to 0.70$)$

0.55 (0.17 to 1.78 )

0.51 (0.15 to 1.70$)$

$0.20(0.02$ to 1.55$)$

$0.47(0.23$ to 0.96$)$

0.32 (0.20 to 0.50$)$

$0.29(0.15$ to 0.58$)$

0.11 (0.03 to 0.38$)$

0.09 (0.01 to 0.69 )

$0.50(0.29$ to 0.86$)$

0.84 (0.28 to 2.55)

0.53 (0.08 to 3.63$)$

0.66 (0.37 to 1.19$)$

$100 \quad 0.43(0.29$ to 0.63$)$

0.42 (0.23 to 0.77$)$

Fig 7 Types of protection against Mycobacterium tuberculosis (TB) in children vaccinated with BCG: protection against infection (irrespective of whether they went on to develop active TB); overall protection against active TB; protection against progression from infection to active TB during screening. $\mathrm{D}+\mathrm{L}=$ DerSimonian and Laird method; $M-\mathrm{H}=\mathrm{Mantel}-\mathrm{Haenszel}$ method 